\title{
Mushroom nutriceuticals in Malaysia
}

\section{Opinion}

Edible mushrooms represent untapped source of nutraceuticals and valuable palatable food. Long viewed as tonics, they are now treasured as functional food that can improve human health and quality of life. Numerous varieties of pharmaceutical substances with potent and unique health-enhancing properties were isolated from medicinal mushrooms and distributed worldwide. They represent a novel class of dietary supplements or nutriceuticals. The two terms have been used interchangeably with functional food, nutraceuticals, phytochemicals, mycochemicals, and biochemopreventatives. Mushroom-based nutriceuticals are products from mycelia or the fruiting bodies of mushrooms, and are consumed in the form of capsules, tablets, or extracts, and have potential therapeutic effects. ${ }^{1}$

Mushroom-based products are being utilized by holistically oriented physicians, herbalists, naturopathic physicians, chiropractors, acupuncturists and Chinese herbal practitioners. Clinical efficacy of mushrooms depends on their precise scope of activity verifiable through in vitro and in vivo studies, dose range, extraction methods, source of substrates, duration and frequency of administration.

Mushrooms have been identified as one of the high-value commodities under Malaysia's National Agro-Food Policy (20112020). Development of the mushroom industry emphasizes on further area expansion, increased productivity, development of new varieties and product quality improvement. At present, three medicinal mushroom varieties, namely the Hericium erinaceus (Bull.: Fr.) Pers., Lignosus rhinocerotis (Cooke) Ryvarden and Ganoderma lucidum (Curtis) P. Karst. are developed as nutriceutical products in Malaysia

Hericium erinaceus, also known as Lion's mane, Monkey's head, Hedgehog mushroom, Satyr's beard, Pom pom blanc, Igelstachelbart, and Yamabushitake is distributed in Asia, Europe and North America. It is a temperate mushroom that requires cool temperatures of $18{ }^{\circ} \mathrm{C}$ to $24^{\circ} \mathrm{C}$ to produce fruiting bodies. Since 2000 , it has been domesticated via adaptation to tropical climate in Malaysia. A standardized aqueous extract from fruiting bodies that contains approximately $21 \% \beta$-(13), (1-6)-glucans and $0.2 \%$ adenosine was marketed as $\mathrm{NevGro}^{\mathrm{TM}}$ by Ganofarm R\&D Sdn Bhd. The mushroom has been extensively tested in in vitro trials in our laboratories using neuronal cell lines, as the neurite outgrowth stimulator in the cultured cells of neural hybrid clone NG108-15 and PC12, and as the regulator of oxidative stress in the dermal fibroblasts of Friedreich's ataxia patients. Further, we explored the ability of locally cultivated $H$. erinaceus in the enhancement of peripheral nerve regeneration and acceleration of motor functional recovery after crush injury. Upregulation of signaling pathways as well as axonal protein synthesis and degradation are consistent with the beginning of motor functional recovery of injured limb. ${ }^{2}$

On the other hand, Lignosus rhinocerotis is a unique National Treasure that has a limited geographical distribution encompassing the Southern China, Thailand, Malaysia, Indonesia, Philippines, Papua New Guinea, New Zealand, and Australia. It is also known as Tiger's milk mushroom or Cendawan susu rimau in Malay language. The indigenous population has been consuming the mushroom as mycomedicine for multi diseases. The freeze-dried cultivated sclerotium marketed as Ligno TM02 ${ }^{\circledR}$ by Ligno Biotech Sdn Bhd,

\author{
Volume II Issue 2 - 2018
}

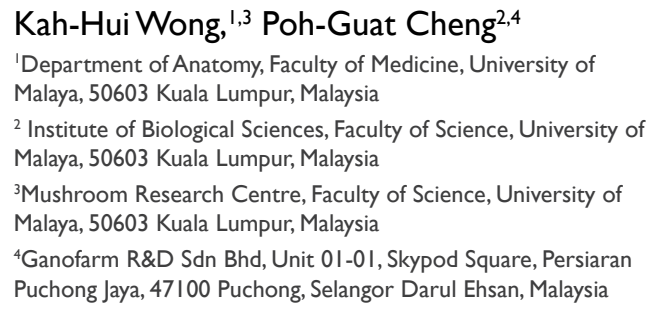

Correspondence: Kah-Hui Wong, Department of Anatomy, Faculty of Medicine, University of Malaya, 50603 Kuala Lumpur, Malaysia, Tel 60379674 729, Fax 60379674 724,

Email wkahhui@um.edu.my

Received: February 24, 20I8| Published: March 26, 2018

were reported to elicit immunomodulation, neuritogenesis ${ }^{3}$ and antineuroinflammatory effects. ${ }^{4}$

Ganoderma lucidum, also known by the Japanese name Reishi and Chinese name Ling zhi, is the most revered mushroom in Traditional Chinese Medicine documented in Shen Nong Ben Cao Jing. Ling zhi is consumed in the form of concoctions by Asian populations for promoting health and longevity. This feature is primarily attributed to polysaccharides and their derivatives. The formulation of $10 \%$ broken spore powder and $90 \%$ concentrated fruiting body powder developed by Ganofarm R\&D Sdn Bhd facilitates bioavailability and absorption in the digestive tract.

Mushroom nutriceuticals as discussed here provide biomaterials with medicinal applications for in vitro and in vivo assessments by targeting multi-mechanisms actions. Therefore, it is essential to establish internationally recognized guidelines for assessing their quality. These products need to be standardized by manufacturing companies. However, a standardized product doesn't necessarily guarantee a product of high quality as there is no legal definition of it. Consumers are often left on their own to decide what is safe and effective for them and the lack of consistent labelling on herbal products can be a source of consumer frustration. Without the implementation of required regulation and reliability in safety and efficacy data, the belief in natural medicines will continue to stagger along.

\section{Acknowledgments}

Fundamental Research Grant Scheme of FP011-2016, High Impact Research Grant UM-MoE UM.C/625/1/HIR/MoE/ASH/01 (H-23001-G000008), High Impact Research Grant UM-MoE UM.C/625/1/HIR/MoE/SC/02 and Science Fund 12-02-03-2050.

\section{Conflict of interest}

Partial sponsorship from Ganofarm R\&D Sdn Bhd for project titled "Neuroprotective activities of NevGro ${ }^{\mathrm{TM}}$ " 


\section{References}

1. Wasser SP, Sokolov D, Reshetnikov SV, et al. Dietary supplements from medicinal mushrooms: Diversity of types and variety of regulations. Int $J$ Med Mushrooms. 2000;2(1):1-19.

2. Wong KH, Gowri K, Phan $\mathrm{CW}$, et al. Restoration of sensory dysfunction following peripheral nerve injury by the polysaccharide from culinary and medicinal mushroom, Hericium erinaceus (Bull.: Fr.) Pers. through its neuroregenerative action. Food Sci Technol (Campinas). 2015;5(4):712721.
3. Seow SLS, Eik LF, Naidu M, et al. Lignosus rhinocerotis (Cooke) Ryvarden mimics the neuritogenic activity of nerve growth factor via MEK/ERK1/2 signaling pathway in PC-12 cells. Sci Rep. 2015;(5):16349.

4. Seow SLS, Naidu M, Sabaratnam V, et al. Tiger's milk medicinal mushroom, Lignosus rhinocerotis (Agaricomycetes) sclerotium inhibits nitric oxide production in LPS-stimulated BV2 microglia. Int $\mathrm{J} \mathrm{Med}$ Mushrooms. 2017;19(5):405-418. 